\title{
A sibling-pair based approach for mapping genetic loci that influence quantitative measures of reading disability
}

\author{
C. Francks, ${ }^{1}$ S. E. Fisher, ${ }^{1}$ A. J. Marlow, ${ }^{1}$ A. J. Richardson, ${ }^{2,3}$ J. F. Stein, ${ }^{2}$ A. P. Monaco ${ }^{1}$ \\ ${ }^{1}$ The Wellcome Trust Centre for Human Genetics, University of Oxford, Oxford, UK \\ ${ }^{2}$ University Laboratory of Physiology, Oxford, UK \\ ${ }^{3} \mathrm{MRI}$ Unit, MRC Clinical Sciences Centre, Imperial College of Medicine, London, UK
}

Summary Family and twin studies consistently demonstrate a significant role for genetic factors in the aetiology of the reading disorder dyslexia. However, dyslexia is complex at both the genetic and phenotypic levels, and currently the nature of the core deficit or deficits remains uncertain. Traditional approaches for mapping disease genes, originally developed for single-gene disorders, have limited success when there is not a simple relationship between genotype and phenotype. Recent advances in high-throughput genotyping technology and quantitative statistical methods have made a new approach to identifying genes involved in complex disorders possible. The method involves assessing the genetic similarity of many sibling pairs along the lengths of all their chromosomes and attempting to correlate this similarity with that of their phenotypic scores. We are adopting this approach in an ongoing genome-wide search for genes involved in dyslexia susceptibility, and have already successfully applied the method by replicating results from previous studies suggesting that a quantitative trait locus at $6 \mathrm{p} 21.3$ influences reading disability. ( 2000 Harcourt Publishers Ltd

\section{INTRODUCTION}

Dyslexia is a condition affecting roughly $5 \%$ of schoolchildren which impairs their ability to read in the absence of any obvious cause such as low intelligence or a lack of educational opportunity. ${ }^{1}$ In recent years it has become clear that dyslexia often represents a broad neurological impairment with effects not confined just to reading problems. Deficits in language syntax can be traced before dyslexic children start to $\mathrm{read}^{2}$ and many dyslexics show delayed speech acquisition and increased rates of speech errors, such as lisps and spoonerisms. ${ }^{3} \mathrm{An}$ inability to correctly process phonemes, the supposed units of mental language processing, is proposed as a core

Received 17 February 2000

Accepted 17 March 2000

Correspondence to: Clyde Francks, The Wellcome Trust Centre for Human Genetics, University of Oxford, Roosevelt Drive, Oxford OX37BN, UK. Tel.: +44 01865 287509; Fax: +44 01865 287501; E-mail: clyde@ well.ox.ac.uk deficit in dyslexia with recent support from functional brain imaging studies of cortical language areas. ${ }^{4}$

More generally, subtle deficits in visual, auditory, and motor pathways have been exposed by brain imaging, evoked potential and psychophysical studies which may point to problems in processing transient stimuli in all modalities. ${ }^{5-8}$ Such deficits can manifest as problems with clumsiness and judging the timing and ordering of events, as well as with poor eye control. These problems may be mediated by neurons with M-cell like properties (with transient, on/off firing) that could represent a family of cell lines using a common developmental mechanism to achieve the correct size and connection pattern. ${ }^{9}$ On a larger scale, brain regions implicated in dyslexia include the language areas of the temporoparietal cortex (reduced left/right asymmetry in dyslexics ${ }^{10}$ ), and the cerebellum, involved in motor and posture control, which may show biochemical asymmetry in dyslexic patients. ${ }^{11}$

The nature of what constitutes the core deficit in dyslexia is therefore still in doubt. With different researchers emphasizing different aspects of the 
phenotype, dyslexia is likely to represent a phenotypically heterogeneous group of disorders. ${ }^{12}$ Castles and Coltheart ${ }^{13}$ pointed to contrasts in the type of reading deficits exhibited by individual dyslexics, and proposed dyslexic subtypes exhibiting either phonological (sub-word) or surface (whole-word) dyslexia in relation to a 'dual route' model of the reading system. Grigorenko et al. ${ }^{14}$ took dissection further by postulating a range of dyslexic subtypes identifiable through tests of different reading related abilities, including phonological awareness (an awareness that words can be deconstructed into phonemic units), phonological decoding (the ability to decode new combinations of phonemes), and single word reading (designed as a measure of overall reading ability). It is these types of reading-based measures which we are currently using in our dyslexia genetic linkage analysis.

\section{GENETIC INVOLVEMENT IN DYSLEXIA}

Evidence for genetic involvement in dyslexia stems from studies documenting familial clustering of the disorder ${ }^{15-19}$ and twin studies which consistently indicate a significant genetic influence. ${ }^{20}$ As a qualitative affection status dyslexia has a $\lambda_{\mathrm{s}}{ }^{21}$ of 12 which is calculated from a sibling risk of $60 \%$ compared with a population prevalence of $5 \%$, although this may vary according to the diagnostic criteria used. Probandwise concordance rates estimated from a large Colorado twin study suggest a $68 \%: 38 \%$ ratio for MZ:DZ twin pairings. ${ }^{22}$ The Colorado study also yielded a heritability of 50-70\% for a quantitative measure incorporating several subtyping scores. ${ }^{23}$

Despite this clear genetic involvement dyslexia does not usually segregate in families as a straightforward single gene disorder. ${ }^{17-19}$ Genetic influences are therefore complex and likely to involve reduced penetrance (where a predisposing allele need not lead to the child developing the disorder), phenocopies (when the disorder develops in individuals who do not have a specific predisposing allele), heterogeneity (when different affected individuals have different genetic backgrounds to their disorders), and oligogenic inheritance (when development of the disorder depends on the transmission and interaction of several different genes). Parametric linkage analysis requires starting assumptions to be made about penetrance, gene frequency, and the inheritance model, and has therefore proved difficult to apply in dyslexia studies given the complexity surrounding both the phenotype itself and the genetic factors involved. The interpretation of results derived from parametric linkage analysis remains uncertain. ${ }^{24-27}$ These studies variously implicated or rejected evidence for linkage to chromosome 15 and chromosome 1p34-36. However, a notable result was recently obtained from a study in which a large
Norwegian pedigree was analyzed under a parametric autosomal dominant transmission model for dyslexia. ${ }^{28}$

\section{Dyslexia and chromosome 6}

Much more consistent success has been achieved using non parametric linkage analysis which avoids misspecifying parameters but instead gains power through large sample sizes. Cardon et al. ${ }^{29,30}$ analyzed two independent sib pair samples using an extension of the DeFries-Fulker regression technique ${ }^{31}$ to establish a quantitative trait locus (QTL) at chromosome 6p21.3 with a composite discriminant score of reading disability $(P=0.0094$ in twins; $P=0.042$ in sib pairs derived from a kindred sample). They focused on the human leukocyte antigen (HLA) region of chromosome $6 p$ because of suggested associations of dyslexia with autoimmune disorders. ${ }^{32,33}$

Grigorenko et al. ${ }^{14}$ used cut-off scores on their phenotypic subtype measures (phoneme awareness, single word reading etc.) to create qualitative affection schemes for each phenotype which they then applied to six extended pedigrees to test for linkage to chromosomes 6,15 and 16. Non parametric affected pedigree member (APM) analysis, ${ }^{34}$ based on allele sharing by affected individuals, showed strongest linkage to the phoneme awareness phenotype on chromosome 6p23-21.3 $\left(P<10^{-6}\right)$. In contrast, traditional parametric analysis yielded a single point linkage $(\mathrm{LOD}=3.15)$ with a marker on chromosome 15 to the single word reading phenotype only. From these results they concluded that different genetic loci influence distinct components of dyslexia susceptibility.

Two subsequent studies of the chromosome $6 \mathrm{p}$ region have each produced evidence for a QTL affecting several different measures of reading disability. ${ }^{35,36}$ The study by Gayan et al. ${ }^{35}$ on a new set of 79 Colorado families with at least two sibs or twins, again using an extension of the DeFries/Fulker regression technique, produced a LOD score of 3.10 across a $5 \mathrm{cM}$ region of $6 \mathrm{p} 22-21.3$ for an 'orthographic coding', or whole word reading phenotype, and $\mathrm{a} \mathrm{LOD}=2.42$ for phonological skills.

Fisher et al..$^{36}$ used a different battery of reading tests, but aimed at similar reading deficits, to study a British sample of 82 families with at least two sibs. Their QTL analysis is based on the assumption that sibs sharing more alleles identical by descent (IBD; see Fig. 1) at a locus influencing a trait will score more similarly on that trait. Fisher et al. ${ }^{36}$ used two complementary statistical approaches to test for this relation, one based on sib differences and the other using variance components. ${ }^{37-39}$ They performed the two types of analysis across several phenotypic measures using both single point and multipoint IBD sharing estimates. All approaches yielded consistent results supporting linkage 

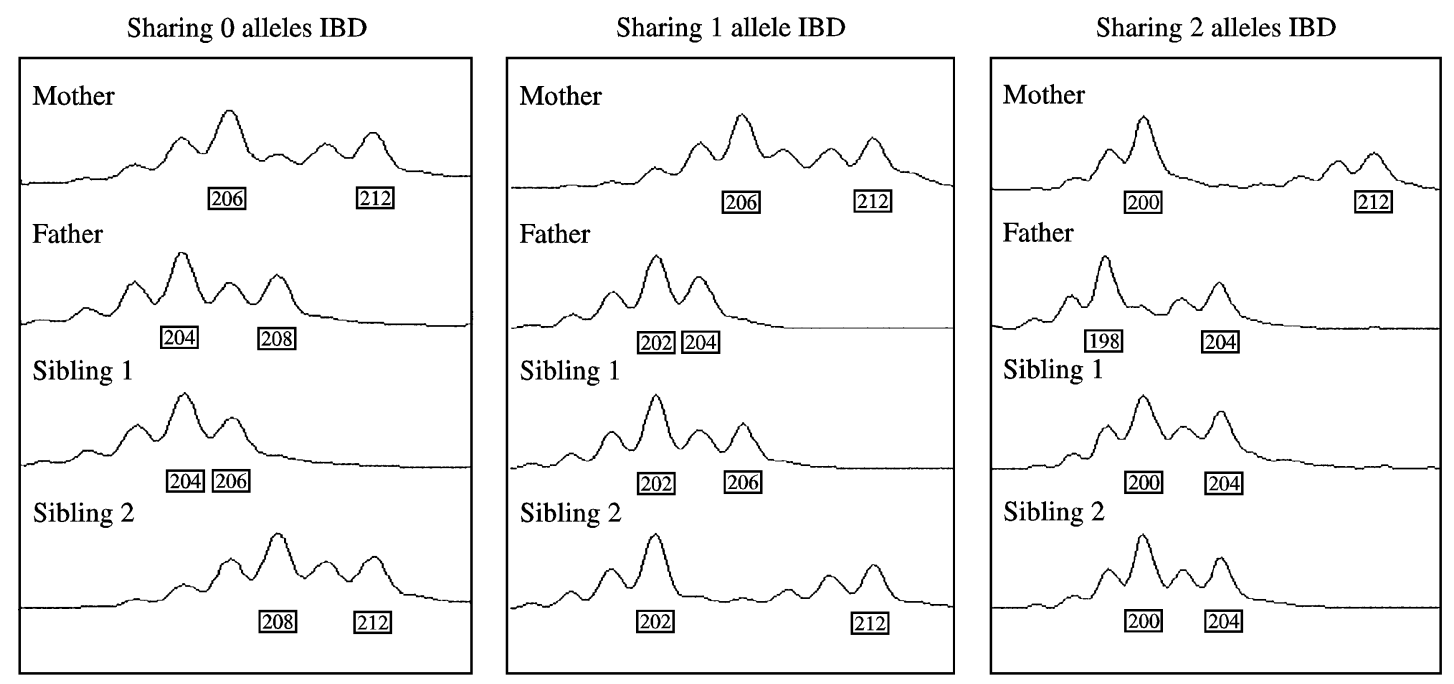

Fig. 1 Sib pair allele sharing detected by fluorescent genotyping in three different families. ${ }^{44}$ Each trace represents a polymorphic marker at the same chromosomal location in all individuals. By using many such markers a picture can be built of the identity-by-descent (IBD) sharing along the entire length of each pair of chromosomes. Our QTL linkage analysis looks for chromosomal regions where there is a correlation between this measure of genetic similarity and the similarity of the psychometric test scores of sibs. Alternatively, linkage disequilibrium analysis tests for associations between specific marker alleles (e.g. the 204 allele above) and reading disability.

of different aspects of reading ability to chromosome 6 p21.3 (sib difference method; irregular words test ( orthographic), $P=0.00035$; nonwords test ( phonological), $P=0.0035$; variance components; irregular words test, $P=0.007$; nonwords test $P=0.0038$ ).

In total, four independent studies have now pointed to chromosome 6p23-21.3 involvement in reading disability, ${ }^{14,29,35,36}$ while one sib pair study did not find significant linkage on chromosome 6 (Field \& Kaplan, ${ }^{40}$ ). The latter study used cut off scores on a measure of phonological impairment to define a qualitative affection status scheme for 'phonological dyslexia'. Failure to find linkage by Field and Kaplan ${ }^{40}$ may support the principle that QTL mapping offers increased power over qualitative affected sib pair analysis for traits which can be measured successfully on a meaningful quantitative scale. Using trait scores directly in analysis also avoids imposing cut off scores for classifying affecteds for which there may be little a priori logic.

\section{Full genome screen}

Success at the chromosome 6 locus suggests that a full genome screen for other QTLs affecting reading disability should be a worthwhile experiment, given that the genetic influences on dyslexia are thought to be oligogenic and heterogeneous, and that the phenotype presents no obvious candidate genes. The chromosome 6 studies demonstrate the power of sib pair QTL methodology to detect loci in a consistent and replicable way across samples ascertained and measured under quite different schemes. However, the precise proportion of total genetic variance attributable to the $6 \mathrm{p}$ QTL is difficult to assess for a number of reasons. ${ }^{36} \mathrm{~A}$ full genome screen will effectively 'zoom out' from this narrow region of interest and allow a better assessment of the strength and significance of the $6 \mathrm{p}$ QTL and others against the broader genetic background of the disorder. No such genome screen has yet been performed.

We now have DNA samples and psychometric test scores from 89 British sib pair families including those of Fisher et al., ${ }^{36}$ and are currently genotyping polymorphic markers at $10 \mathrm{cM}$ intervals across all chromosomes for all samples. We will analyze the data from the genome scan using quantitative methods including some similar to those used in Fisher et al., ${ }^{36}$ which continue to be extended and improved. In addition, we have an ongoing collaboration with the Colorado dyslexia study and are currently genotyping $119 \mathrm{sib}$ or twin pair families collected there.

\section{From linkage to gene}

The chromosomal locations of interest that sib pair linkage analysis yields are inevitably very large. The challenge following the genome screen will be to narrow down the regions enough to begin physical mapping or candidate gene studies with confidence. We will attempt to increase our power and resolution at loci produced by the initial linkage screen through the application of 
linkage disequilibrium methods in which specific alleles are tested for an association with the disorder. It will soon be possible to achieve the required density of markers for detecting linkage disequilibrium (estimated at 1 marker every $6 \mathrm{~kb}^{41}$ ) by identifying common single nucleotide polymorphisms (SNPs) which occur on average every $1 \mathrm{~kb}$ throughout the genome. ${ }^{42}$ The SNP Consortium ${ }^{43}$ aims to generate 300000 SNPs over the next 2 years, which will create a starting map with SNPs at an average spacing of $20 \mathrm{~kb}$.

The eventual goal of our research into the genetics of dyslexia is to find functional polymorphisms of genes associated with dyslexia susceptibility. Once we have narrowed down regions of interest sufficiently to begin screening candidate genes, we will be guided in our choice of candidates by functional hypotheses. One such guiding hypothesis will be the proposition that fatty acid metabolism may be disrupted in dyslexic patients. The challenge will then be to characterize functionally the genes and the effects of polymorphisms that we find through expression and subcellular localization studies, and the use of animal models. The discovery of genes involved in reading disability will create new possibilities for the early diagnosis and treatment of dyslexia, and will also allow a new insight into the development and functioning of information processing systems in the brain.

\section{ACKNOWLEDGEMENTS}

We are very grateful to all the families who are participating in our dyslexia study. We thank Janet Walter, Pan Southcott and Sue Fowler for their continued effort in collecting blood samples and administering psychometric tests.

This project is supported by the Wellcome Trust. A. P. Monaco is a Wellcome Trust Principal Research Fellow. C. Francks is a Wellcome Trust Prize Student.

\section{REFERENCES}

1. Shaywitz S. E., Shaywitz B. A., Fletcher J. M., Escobar M. D. Prevalence of reading disability in boys and girls. JAMA 1990; 264: 998-1002.

2. Scarborough H. S. Very early language deficits in dyslexic children. Child Dev 1990; 61: 1728-1743.

3. Pennington B. F. Reading disabilities: genetic and neurological influences. Boston: Kluwer Academic Press, 1991.

4. Shaywitz S. E., Shaywitz B. A., Pugh K. R., et al. Functional disruption in the organization of the brain for reading in dyslexia. Pro Natl Acad Sci USA 1998; 95: 2636-2641.

5. Stein J. Developmental dyslexia, neural timing, and hemispheric lateralisation. Int J Psychophysiology 1994; 570: 971-980.

6. Stein J, Walsh V. To see but not to read: the magnocellular theory of dyslexia. Trends Neurosci 1997; 20: 147-152.
7. Eden G. F., VanMeter J. W., Rumsey J. M., Maisog J. M., Woods R. P., Zeffiro T. A. Abnormal processing of visual motion in dyslexia revealed by functional brain imaging. Nature 1996; 382: 66-69.

8. Demb J. B., Boynton G. M., Best M., Heeger D. J. Psychophysical evidence for a magnocellular pathway deficit in dyslexia. Vision Res 1998; 38: 1555-1559.

9. Stein J. F., Talcott J. B. Impaired neuronal timing in developmental dyslexia-the magnocellular hypothesis. Dyslexia 1999; 5: 59-78.

10. Galaburda A. M. Dyslexia and development. Harvard University Press, 1993.

11. Rae C., Lee M. A., Dixon R. M. et al. Metabolic abnormalities in developmental dyslexia detected by $1^{\mathrm{H}}$ magnetic resonance spectroscopy. Lancet 1998; 351: 1849-1852.

12. Smith S. D., Gilger J. W., Pennington B. F. Dyslexia and other specific learning disorders. In: Rimoin D. L., Connor J. M., Pyeritz R. E., eds. Principles and practice of medical genetics. New York: Churchill Livingston, 1996; 1767-1789.

13. Castles A., Coltheart M. Varieties of developmental dyslexia. Cognition 1993; 47: 149-180.

14. Grigorenko E. L., Wood F. B., Meyer M. S., et al. Susceptibility loci for distinct components of developmental dyslexia on chromosomes 6 and 15. Am J Hum Genet 1997; 60: 27-39.

15. Thomas C. J. Congenital 'word-blindness' and its treatment Ophthalmoscope 1905; 3: 380-385.

16. Hallgren B. Specific dyslexia (congenital word blindness): a clinical and genetic study. Acta Psychiatr Neurol 1950; 65: 2-289.

17. Lewitter F. I., DeFries J. C., Elston R. C. Genetic models of reading disabilities. Behav Genet 1980; 10: 9-30.

18. Pennigton B. F., Gilger J. W., Pauls D., Smith S. A., Smith S. D., DeFries J. C. Evidence for major gene transmission of developmental dyslexia. JAMA 1991; 266: 1527-1534.

19. Lubs H. A., Rabin M., Feldman E., et al. Familial dyslexia: genetic and medical findings in eleven three-generation families. Ann Dyslexia 1993; 43: 44-60.

20. DeFries J. C., Fulker D. W., LaBuda M. C. Evidence for a genetic aetiology in reading disability of twins. Nature 1987; 329: 537-539.

21. Risch N. Linkage strategies for complex traits. I. Multilocus models. II. The power of affected relative pairs. III. The effect of marker polymorphism on analysis of affected relative pairs. $A m \mathrm{~J}$ Hum Genet 1990; 46: 222-253.

22. DeFries J. C., Alarcon M. Genetics of specific reading disability. Mental Retard Dev Disabilities Res Rev 1996; 2: 39-47.

23. DeFries J. C., Gillis J. J. Genetics of reading disability. In: Plomin R., McClearn G. eds. Nature, nurture, and psychology. Washington, DC, APA Press, 1993; 121-145.

24. Smith S. D., Kimberling W. J., Pennington B. F., Lubs H. A. Specific reading disability: identification of an inherited form through linkage analysis. Science 1983; 219: 1345-1347.

25. Bisgaard M. L., Eiberg H., Moller N., Niebuhr E., Mohr J. Dyslexia and chromosome 15 heteromorphism: negative LOD score in a Danish sample. Clin Genet 1987; 32: 118-119.

26. Froster U., Schulte-Korne G., Hebebrand J., Remschmidt H. Cosegregation of a balanced translocation $(1: 2)$ with retarded speech development and dyslexia. Lancet 1993; 342: 178-179.

27. Rabin M., Wen X. L., Hepburn M., Lubs H. A., Feldman E., Duara R. Suggestive linkage of developmental dyslexia to chromosome 1p34-p36. Lancet 1993; 342: 178.

28. Fagerheim T., Raeymaekers P., Toenessen F. E., Pedersen M., Tranebjaerg L., Lubs H. A. A new gene (DYX3) for dyslexia is located on chromosome 2. J Med Genet 1999; 36: 664-669.

29. Cardon L. R., Smith S. D., Fulker D. W., Kimberling W. J., Pennington B. F., DeFries J. C. Quantitative trait locus for 
reading disability on chromosome 6 . Science $1994 ; \mathbf{2 6 8}$ : 276-279.

30. Cardon L. R., Smith S. D., Fulker D. W., Kimberling W. J., Pennington B. F., DeFries J. C. Quantitative trait locus for reading disability: correction. Science 1995; 268: 1553.

31. DeFries J. C., Fulker D. W. Multiple regression analysis of twin data. Behav Genet 1985; 15: 467-473.

32. Pennington B. F., Smith S., Kimberling W., Green P., Haith M. Lefthandedness and immune disorders in familial dyslexics. Arch Neurol 1987; 44: 634-639.

33. Hugdahl K., Synnevag B., Satz P. Immune and autoimmune disorders in dyslexic children. Neuropsychologia 1990; 28: 673-679.

34. Weeks D. E., Lange K. The affected-pedigree-member method of linkage analysis. Am J Hum Genet 1988; 42: 315-326.

35. Gayan J., Smith S. D., Cherny S. S., et al. Quantitative-trait locus for specific language and reading deficits on chromosome 6p. Am J Hum Genet 1999; 64: 157-164.

36. Fisher S. E., Marlow A. J., Lamb J., et al. A quantitative trait locus on chromosome $6 \mathrm{p}$ influences different aspects of developmental dyslexia. Am J Hum Genet 1999; 64: 146-156.
37. Haseman J. K., Elston R. C. The investigation of linkage between a quantitative trait and a marker locus. Behav Genet 1972; 2: 3-19.

38. Kruglyak L., Lander E. S. Complete multipoint sib-pair analysis of qualitative and quantitative traits. Am J Hum Genet 1995; 57 439-454.

39. Amos C. I. Robust variance components approach for assessing genetic linkage in pedigrees. Am J Hum Genet 1994; 54: 535-543.

40. Field L. L., Kaplan B. J. Absence of linkage of phonological coding dyslexia to chromosome $6 \mathrm{p} 23-\mathrm{p} 21.3$ in a large family data set. $A m$ J Hum Genet 1998; 63: 1448-1456.

41. Kruglyak L. Prospects for whole-genome linkage disequilibrium mapping of common disease genes. Nature Genetics 1999; 22: 139-144.

42. Risch N., Merikangas K. The future of genetic studies of complex human diseases. Science 1996; 273: 1516-1517.

43. http://snp.cshl.org/index.html

44. Fisher S. E., Stein J. F., Monaco A. P. A genome wide search strategy for identifying quantitative trait loci involved in reading and spelling disability (developmental dyslexia). Eur Child Adolesc Psychiatry 1999; 8: S3, 47-51. 